EPiC Series in Computing
Volume 61, 2019, Pages 14-40
$\begin{gathered}\text { ARCH19. 6th International Workshop on Applied } \\ \text { Verification of Continuous and Hybrid Systems }\end{gathered}$

\title{
ARCH-COMP19 Category Report: Continuous and Hybrid Systems with Linear Continuous Dynamics
}

\author{
Matthias Althoff ${ }^{1}$, Stanley Bak ${ }^{2}$, Marcelo Forets ${ }^{3}$, Goran Frehse ${ }^{4}$, Niklas \\ Kochdumper $^{1}$, Rajarshi Ray ${ }^{5}$, Christian Schilling ${ }^{6}$, and Stefan Schupp ${ }^{7}$ \\ 1 Technische Universität München, Department of Informatics, Munich, Germany \\ althoff@in.tum.de \\ 2 Safe Sky Analytics, Manlius, NY, United States \\ stanleybak@gmail.com \\ 3 UTEC Universidad Tecnológica, Uruguay \\ marcelo.forets@utec.edu.uy \\ 4 ENSTA ParisTech, Palaiseau, France \\ goran.frehse@ensta-paristech.fr \\ 5 National Institute of Technology Meghalaya, Shillong, India. \\ rajarshi.ray@nitm.ac.in \\ 6 IST Austria, Klosterneuburg, Austria \\ christian.schilling@ist.ac.at \\ 7 RWTH Aachen University, Theory of hybrid systems, Aachen, Germany \\ stefan.schupp@cs.rwth-aachen.de
}

\begin{abstract}
This report presents the results of a friendly competition for formal verification of continuous and hybrid systems with linear continuous dynamics. The friendly competition took place as part of the workshop Applied Verification for Continuous and Hybrid Systems $(\mathrm{ARCH})$ in 2019. In its third edition, seven tools have been applied to solve six different benchmark problems in the category for linear continuous dynamics (in alphabetical order): CORA, CORA/SX, HyDRA, Hylaa, JuliaReach, SpaceEx, and XSpeed. This report is a snapshot of the current landscape of tools and the types of benchmarks they are particularly suited for. Due to the diversity of problems, we are not ranking tools, yet the presented results provide one of the most complete assessments of tools for the safety verification of continuous and hybrid systems with linear continuous dynamics up to this date.
\end{abstract}




\section{Introduction}

Disclaimer The presented report of the ARCH friendly competition for continuous and hybrid systems with linear continuous dynamics aims at providing a landscape of the current capabilities of verification tools. We would like to stress that each tool has unique strengths - not all of the specificities can be highlighted within a single report. To reach a consensus in what benchmarks are used, some compromises had to be made so that some tools may benefit more from the presented choice than others. The obtained results have been verified by an independent repeatability evaluation. To establish further trustworthiness of the results, the code with which the results have been obtained is publicly available at gitlab.com/goranf/ARCH-COMP.

This report summarizes results obtained in the 2019 friendly competition of the ARCH workshop $^{1}$ for verifying hybrid systems with linear continuous dynamics

$$
\dot{x}(t)=A x(t)+B u(t),
$$

where $A \in \mathbb{R}^{n \times n}, x \in \mathbb{R}^{n}, B \in \mathbb{R}^{n \times m}$, and $u \in \mathbb{R}^{m}$. Participating tools are summarized in Sec. 2. The results of our selected benchmark problems are shown in Sec. 3 and are obtained on the tool developers' own machines. Thus, one has to factor in the computational power of the processors used, summarized in Appendix A, as well as the efficiency of the programming language of the tools.

The goal of the friendly competition is not to rank the results, but rather to present the landscape of existing solutions in a breadth that is not possible with scientific publications in classical venues. Such publications would typically require the presentation of novel techniques, while this report showcases the current state-of-the-art tools. For all results reported by each participant, we have run an independent repeatability evaluation. A novelty compared to last year is that tool developers provide Dockerfiles [13] so that executing tools on one's own machine has become much easier.

The selection of the benchmarks has been conducted within the forum of the ARCH website (cps-vo.org/group/ARCH), which is visible for registered users and registration is open for anybody. All tools presented in this report use some form of reachability analysis. This, however, is not a constraint set by the organizers of the friendly competition. We hope to encourage further tool developers to showcase their results in future editions.

\section{Participating Tools}

The tools participating in the category Continuous and Hybrid Systems with Linear Continuous Dynamics are introduced subsequently in alphabetical order.

CORA The tool COntinuous Reachability Analyzer (CORA) [1, 3, 4] realizes techniques for reachability analysis with a special focus on developing scalable solutions for verifying hybrid systems with nonlinear continuous dynamics and/or nonlinear differential-algebraic equations. A further focus is on considering uncertain parameters and system inputs. Due to the modular

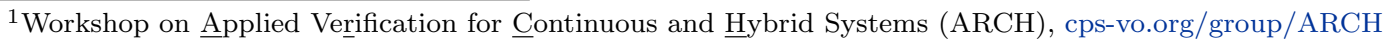


design of CORA, much functionality can be used for other purposes that require resourceefficient representations of multi-dimensional sets and operations on them. CORA is implemented as an object-oriented MATLAB code. The modular design of CORA makes it possible to use the capabilities of the various set representations for other purposes besides reachability analysis. CORA is available at cora.in.tum.de.

CORA/SX CORA/SX is a port of the basic zonotope reachability algorithm from the CORA MATLAB toolbox to SpaceEx. It varies slightly in that some matrix computations (which approximate the input over one time step) use SpaceEx code instead of an over-approximation that is based on intervals.

HyDRA The Hybrid systems Dynamic Reachability Analysis (HyDRA) tool implements flow-pipe construction based reachability analysis for linear hybrid automata. The tool is built on top of HyPro [26] available at ths.rwth-aachen.de/research/projects/hypro/, a C++ library for reachability analysis. HyPro provides different implementations of set representations tailored for reachability analysis such as boxes, convex polyhedra, support functions, or zonotopes, all sharing a common interface. This interface allows one to easily exchange the utilized set representation in HyDRA. We use this to extend state-of-the art reachability analysis by CEGAR-like parameter refinement loops, which (among other parameters) allow us to vary the used set representation. Furthermore, HyDRA incorporates the capability to explore different branches of the search tree in parallel. Being in an early state of development, HyDRA already shows promising results on some benchmarks, although there is still room for improvements. An official first release is planned.

Hylaa The tool Hylaa $[9,10]$ computes the reachable set using discrete-time semantics. Hylaa is a Python-based tool, which can produce live plots during computation, as well as images and video files of the reachable set. Hylaa's website is http://stanleybak.com/hylaa.

The biggest differences with discrete-time semantics is that safety is not checked between time steps, and time-varying inputs are constant between time steps, rather than considering all possible input frequencies [11]. The benefit of this is that Hylaa's analysis is exact with respect to discrete-time semantics and it can always generates counter-example when specifications are violated. Note that continuous-time and discrete-time semantics are incomparable, neither one is strictly contained in the other. However, when small time steps are used, the results are qualitatively similar, as can be seen from some of the reach set plots in this year's competition.

We used a newer implementation of Hylaa, version 2, where we removed some of the previous version's optimization in order to generalize to more complex dynamics (resets and time-varying inputs across discrete transitions). The result, if you compare this year's numbers with last year's, is that the tool is slightly slower on the large continuous systems, but we are able to analyze more benchmarks including platoon, gearbox, and more complex versions of spacecraft rendezvous. Further, Hylaa v2 has a new feature where aggregated states across discrete transitions can be later split, in order to maintain the exactness of the analysis (aggregation typically adds overapproximation error). Earlier versions of this were present before, but those required full deaggregation as soon as a guard was reached, which scales exponentially with respect to the number of discrete jumps. The newer version less aggressive about when performing deaggregation, which was needed for all the instances of the spacecraft rendezvous benchmark. A publication outlining the new technique is currently under preparation. 
JuliaReach JuliaReach is a toolbox for reachability computations of dynamical systems [14], available at http://juliareach.org. It is written in Julia, a modern high-level language for scientific computing. Compared to last year, JuliaReach now has support for hybrid systems (and also nonlinear dynamics). The continuous-time reachability algorithm for linear systems uses a block decomposition technique based on support functions presented in [15]. This technique partitions the state space, projects the initial states to subspaces, and propagates these low-dimensional sets in time. This allows us to perform otherwise expensive set operations in low dimensions. Furthermore, if the output does not depend on all dimensions, we can effectively skip the reach set computation for the respective dimensions. For checking safety properties in purely continuous time, we only compute the reachable states symbolically, which allows us to make the minimal number of support-function evaluations. JuliaReach also offers an option to analyze systems with discrete-time semantics as in Hylaa. For the set computations we use the LazySets library, which is also part of the JuliaReach toolbox. For some of the models we use our custom $S X$ parser for parsing SX (SpaceEx format) model files, and otherwise we create the models manually in Julia. For next year we plan to extend the toolbox with complementary techniques and algorithms to make it more effective, which is now easy with our new architecture.

SpaceEx SpaceEx is a tool for computing reachability of hybrid systems with complex, highdimensional dynamics $[20,21,19]$. It can handle hybrid automata whose continuous and jump dynamics are piecewise affine with nondeterministic inputs. Nondeterministic inputs are particularly useful for modeling the approximation error when nonlinear systems are brought to piecewise affine form. SpaceEx comes with a web-based graphical user interface and a graphical model editor. Its input language facilitates the construction of complex models from automata components that can be combined to networks and parameterized to construct new components. The analysis engine of SpaceEx combines explicit set representations (polyhedra), implicit set representations (support functions) and linear programming to achieve a maximum of scalability while maintaining high accuracy. It constructs an overapproximation of the reachable states in the form of template polyhedra. Template polyhedra are polyhedra whose faces are oriented according to a user-provided set of directions (template directions). A cover of the continuous trajectories is obtained by time-discretization with an adaptive time-step algorithm. The algorithm ensures that the approximation error in each template direction remains below a given value. SpaceEx is available at http://spaceex.imag.fr.

XSpeed The tool XSpeed implements algorithms for reachability analysis for continuous and hybrid systems with linear dynamics. The focus of the tool is to exploit the modern multicore architectures and enhance the performance of reachability analysis through parallel computations. XSpeed realizes two algorithms to enhance the performance of reachability analysis of purely continuous systems. The first is the parallel support function sampling algorithm and the second is the time-slicing algorithm [24, 25]. The performance of hybrid systems reachability analysis is enhanced using an adaptation of the G.J. Holzmann's parallel BFS algorithm in the SPIN model checker, called the AGJH algorithm [23]. In addition, a task parallel and an asynchronous variant of AGJH are also implemented in the tool. XSpeed is available at http://xspeed.nitmeghalaya.in/ 


\section{Verification of Benchmarks}

For the 2019 edition, we have decided to keep all benchmarks from our 2018 friendly competition [2], but solve them with our updated tools. In addition, we have modified the Spacecraft Rendezvous benchmark and the Gearbox benchmark. In the Spacecraft Rendezvous benchmark we have increased the uncertainty of the time of the switch to the passive (abort) mode, which can be significantly more challenging to verify. Regarding the Gearbox benchmark, we have added an instance with a larger initial set.

Special Features We briefly list the special features of each benchmark:

- Space station benchmark from [27]: This is a purely continuous benchmark with 270 state variables and three inputs. This year, it is the benchmark with the largest amount of continuous state variables.

- Spacecraft Rendezvous benchmark from [16]: This benchmark has hybrid dynamics and is a linearization of a benchmark in the other ARCH-COMP category Continuous and Hybrid Systems with Nonlinear Dynamics. Consequently, the reader can observe the difference in computation time and verification results between the linearized version and the original dynamics.

- Powertrain benchmark from [5, Sec. 6]: This is a hybrid system for which one can select the number of continuous state variables and the size of the initial set. Up to 51 continuous state variables are considered.

- Building benchmark from [27, No. 2]: A purely continuous linear system with a medium number of continuous state variables; the benchmark does not only have safety properties, but also ones that should be violated to check whether the reachable sets contain certain states.

- Platooning benchmark from [12]: A rather small number of continuous state variables is considered, but one can arbitrarily switch between two discrete states: a normal operation mode and a communication-failure mode.

- Gearbox benchmark from [17]: This benchmark has the smallest number of continuous state variables, but the reachable set does not converge to a steady state and the reachable set for one point in time might intersect multiple guards at once.

Types of Inputs Generally, we distinguish between three types of inputs:

1. Fixed inputs, where $u(t)$ is precisely known. If in addition, $u(t)=$ const, the linear system becomes an affine system $\dot{x}(t)=A x(t)+b$. For instance, the gearbox benchmark has affine dynamics.

2. Uncertain but constant inputs, where $u(t) \in \mathcal{U} \subset \mathbb{R}^{m}$ is uncertain within a set $\mathcal{U}$, but each uncertain input is constant over time: $u(t)=$ const.

3. Uncertain, time-varying inputs $u(t) \in \mathcal{U} \subset \mathbb{R}^{m}$ where $u(t) \neq$ const. Those systems do not converge to a steady state solution and consider uncertain inputs of all frequencies. For tools that cannot consider arbitrarily varying inputs, we have stated that changes in inputs are only considered at fixed points in time. 
Different Paths to Success When tools use a fundamentally different way of solving a benchmark problem, we add further explanations.

Computation Time The computation times specified in this report include the computation time of the reachable set and the time needed for the verification of the specifications.

\subsection{International Space Station Benchmark}

\subsubsection{Model}

The International Space Station (ISS) is a continuous linear time-invariant system $\dot{x}(t)=$ $A x(t)+B u(t)$ proposed as a benchmark in ARCH 2016 [27]. In particular, the considered system is a structural model of component 1R (Russian service module), which has 270 state variables with three inputs.

The specification deals with the possible range for output $y_{3}$, which is a linear combination of the state variables $\left(y=C x, C \in \mathbb{R}^{3 \times 270}\right)$. Initially all 270 variables are in the range $[-0.0001,0.0001], u_{1}$ is in $[0,0.1], u_{2}$ is in $[0.8,1]$, and $u_{3}$ is in $[0.9,1]$. The time bound is 20 . Discrete-time analysis for the space station benchmark should be done with a step size of 0.01 . The A, B, and C matrices are available in MATLAB format ${ }^{2}$ (that can also be opened with Python using scipy.io.loadmat) and in SpaceEx format ${ }^{3}$. There are two versions of this benchmark:

ISSF01 The inputs can change arbitrarily over time: $\forall t: u(t) \in \mathcal{U}$.

ISSC01 (constant inputs) The inputs are uncertain only in their initial value, and constant over time: $u(0) \in \mathcal{U}, \dot{u}(t)=0$.

\subsubsection{Specifications}

The verification goal is to check the ranges reachable by the output $y_{3}$, which is a linear combination of the state variables. In addition to the safety specification, for each version there is an UNSAT instance that serves as sanity checks to ensure that the model and the tool work as intended. But there is a caveat: In principle, verifying an UNSAT instance only makes sense formally if a witness is provided (counter-example, under-approximation, etc.). Since most of the participating tools do not have this capability, we run the tools with the same accuracy settings on an SAT-UNSAT pair of instances. The SAT instance demonstrates that the over-approximation is not too coarse, and the UNSAT instance demonstrates that the over-approximation is indeed conservative, at least in the narrow sense of the specification.

ISS01 Bounded time, safe property: For all $t \in[0,20], y_{3}(t) \in[-0.0007,0.0007]$. This property is used with the uncertain input case (ISSF01) and assumed to be satisfied.

ISS02 Bounded time, safe property: For all $t \in[0,20], y_{3}(t) \in[-0.0005,0.0005]$. This property is used with the constant input case (ISSC01) and assumed to be satisfied.

ISU01 Bounded time, unsafe property: For all $t \in[0,20], y_{3}(t) \in[-0.0005,0.0005]$. This property is used with the uncertain input case (ISSF01) and assumed to be unsatisfied.

ISU02 Bounded time, unsafe property: For all $t \in[0,20], y_{3}(t) \in[-0.00017,0.00017]$. This property is used with the constant input case (ISSC01) and assumed to be unsatisfied.

\footnotetext{
${ }^{2}$ slicot.org/objects/software/shared/bench-data/iss.zip

${ }^{3}$ cps-vo.org/node/34059
} 


\subsubsection{Results}

Results of the international space station benchmark for state $y_{3}$ over time are shown in Fig. 1 and Fig. 2. The computation times of various tools for the benchmark are listed in Tab. 1.

Note CORA CORA was run with a step size of 0.01 and with a zonotope order of 30 for benchmark version ISSF01. For version ISSC01 a step size of 0.02 and a zonotope order of 10 was used.

Note JuliaReach We used the following step sizes (in dense time): 0.0006 for ISSF01 and 0.005 for ISSC01. Moreover, we handled inputs in a wrapping-free way. The matrix exponential obtained from the discretization is very sparse, which we can exploit in our decomposition-based approach. For the property we need to compute (symbolic) reachable states for 135 variables. We used a two-block partition with each block consisting of 135 variables.

Note SpaceEx SpaceEx was run with the LGG scenario. The sampling was chosen as 0.005 for ISSF01 and 0.05 for ISSC01. The template directions were taken to be $\pm y_{3}$, so only two directions. Since $y_{3}$ is an algebraic variable that is a linear expression of the state variables, we replaced it in the forbidden states and the direction definition by the corresponding linear expression. To model the constant inputs in ISSC01, we introduced $u_{1}, u_{2}, u_{3}$ as state variables with $\dot{u}_{1}=\dot{u}_{2}=\dot{u}_{3}=0$. A custom algorithm for constant inputs could avoid such an artificial augmentation and significantly reduce the runtime for ISSC01. Note that SpaceEx treats the initial states as a general polyhedron, i.e., a linear program is solved at every time step. SpaceEx also computes the full matrix exponential, a $270 \times 270$ matrix, even though in the LGG algorithm it would suffice to compute the vector $e^{A t} \ell$ for each template direction $\ell$.

Since SpaceEx does not currently support the plotting of algebraic variables, we used the following trick to plot $y_{3}$ over time: we introduced a state variable $z$ with dynamics $\dot{z}=$ $-1000\left(z-y_{3}\right)$. Since the time constant for $z$ is about two orders of magnitude below that of $y_{3}$, we expect the plots to practically identical to a true plot of $y_{3}$.

Table 1: Computation Times for the International Space Station Benchmark

\begin{tabular}{|c|c|c|c|c|c|c|}
\hline \multirow[b]{3}{*}{ tool } & \multicolumn{4}{|c|}{ computation time in $[\mathrm{s}]$} & \multicolumn{2}{|c|}{ platform } \\
\hline & \multicolumn{2}{|c|}{ ISSF01 } & \multicolumn{2}{|c|}{ ISSC01 } & \multirow[b]{2}{*}{ language } & \multirow{2}{*}{$\begin{array}{l}\text { machine } \\
\text { (Sec. A) }\end{array}$} \\
\hline & ISS01 & ISU01 & ISS02 & ISU02 & & \\
\hline CORA & 253 & 149 & 6 & 0.19 & MATLAB & $\mathrm{M}_{\mathrm{CORA}}$ \\
\hline JuliaReach & 2.4 & 1.3 & 0.5 & 0.3 & Julia & $\mathrm{M}_{\text {JuliaReach }}$ \\
\hline SpaceEx & 36.4 & 39.3 & 24.7 & 24.3 & $\mathrm{C}++$ & $\mathrm{M}_{\text {SpaceEx }}$ \\
\hline \multicolumn{7}{|c|}{ discrete-time tools } \\
\hline Hylaa & 401 & 109 & 60 & 0.89 & Python & $\mathrm{M}_{\text {Hylaa }}$ \\
\hline JuliaReach & 0.5 & 2.2 & 0.5 & 0.1 & Julia & $\mathrm{M}_{\text {JuliaReach }}$ \\
\hline
\end{tabular}




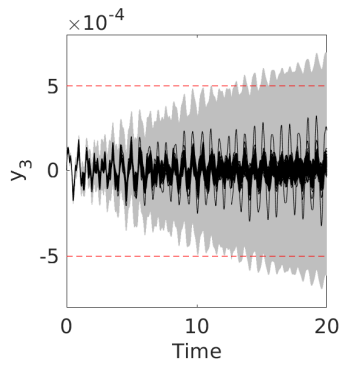

(a) CORA.

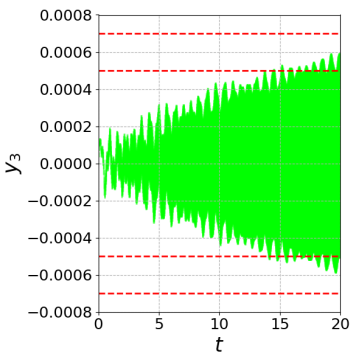

(b) Hylaa.

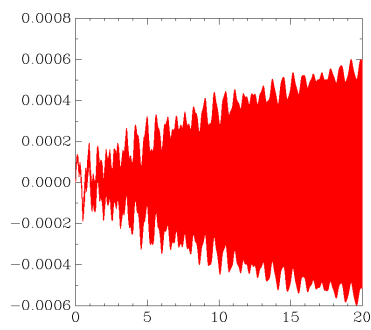

(d) SpaceEx.

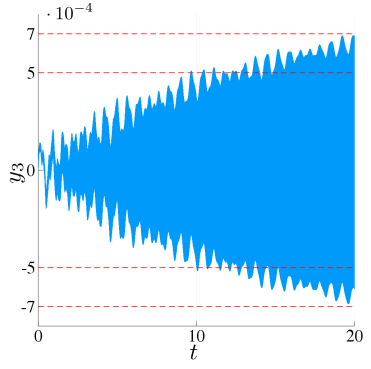

(c) JuliaReach

Figure 1: ISS: Reachable sets of $y_{3}$ plotted over time for the uncertain input case.

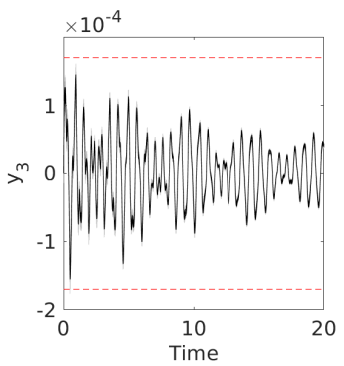

(a) CORA.

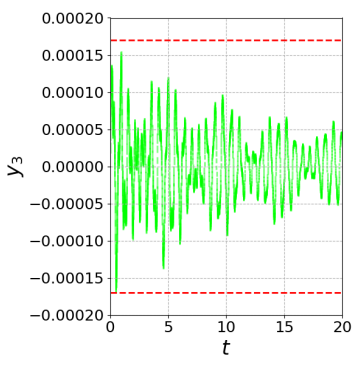

(b) Hylaa.

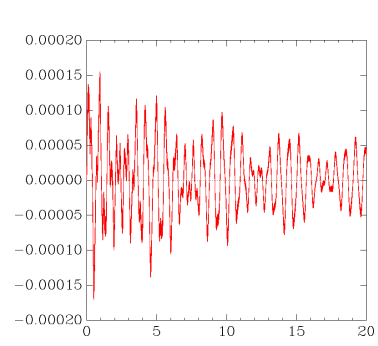

(d) SpaceEx.

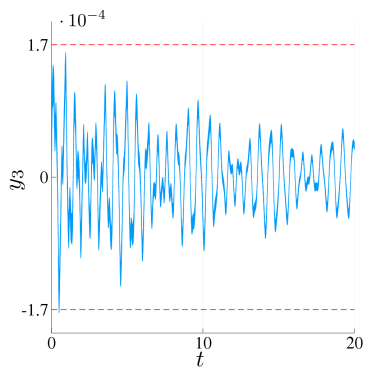

(c) JuliaReach.

Figure 2: ISS: Reachable sets of $y_{3}$ plotted over time for the constant input case. 


\subsection{Spacecraft Rendezvous Benchmark}

\subsubsection{Model}

Spacecraft rendezvous is a perfect use case for formal verification of hybrid systems since mission failure can cost lives and is extremely expensive. This benchmark is taken from [16]; its original continuous dynamics is nonlinear, and the original system is verified in the ARCHCOMP category Continuous and Hybrid Systems with Nonlinear Dynamics. When spacecraft are in close proximity (such as rendezvous operations), a common approximation to analyze the nonlinear dynamics is to use the linearized Clohessy-Wiltshire-Hill (CWH) equations [18]. This benchmark analyzes this linear hybrid model.

The hybrid nature of this benchmark originates from a switched controller, while the dynamics of the spacecraft is purely continuous. In particular, the modes are approaching (100m$1000 \mathrm{~m}$ ), rendezvous attempt (less than 100m), and aborting. Discrete-time analysis for the rendezvous system should be done with a step size of 0.1 . The model is available in C2E2, SDVTool, and SpaceEx format on the ARCH website ${ }^{4}$. The set of initial states is

$$
\mathcal{X}_{0}=\left[\begin{array}{c}
-900 \\
-400 \\
0 \\
0
\end{array}\right] \oplus\left[\begin{array}{c}
{[-25,25]} \\
{[-25,25]} \\
0 \\
0
\end{array}\right]
$$

In previous years, there were two versions of this benchmark:

SRNA01 The spacecraft approaches the target as planned and there exists no transition into the aborting mode.

SRA01 A transition into aborting mode occurs at time $t=120$ [min].

This year, we have added additional instances that transition to the aborting mode at different times, in order to increase the analysis difficulty and add unsafe cases. This required increasing the analysis time horizon to 300 minutes. The additional instances are as follows.

SRA02 A transition into aborting mode occurs nondeterministically, $t \in[120,125]$ [min].

SRA03 A transition into aborting mode occurs nondeterministically, $t \in[120,145]$ [min].

SRA04 A transition into aborting mode occurs at time $t=240$ [min].

SRA05 A transition into aborting mode occurs nondeterministically, $t \in[235,240]$ [min].

SRA06 A transition into aborting mode occurs nondeterministically, $t \in[230,240]$ [min].

SRA07 A transition into aborting mode occurs nondeterministically, $t \in[50,150]$ [min].

SRA08 A transition into aborting mode occurs nondeterministically, $t \in[0,240]$ [min].

An initial, discrete-time analysis indicated it is safe to enter the aborting mode up to around time $t=250$ [min]. We also added the following two instances, which are presumably unsafe. For timing, tools should use the same settings for these as for the safe cases.

SRU01 A transition into aborting mode occurs at time $t=260$ [min].

SRU02 A transition into aborting mode occurs nondeterministically, $t \in[0,260]$ [min].

\footnotetext{
${ }^{4}$ cps-vo.org/node/36349
} 


\subsubsection{Specifications}

Given the thrust constraints of the specified model, in mode rendezvous attempt, the absolute velocity must stay below $0.055 \mathrm{~m} / \mathrm{s}$. In the aborting mode, the vehicle must avoid the target, which is modeled as a box $\mathcal{B}$ with $0.2 \mathrm{~m}$ edge length and the center placed as the origin. In the rendezvous attempt the spacecraft must remain within the line-of-sight cone $\mathcal{L}=\left\{[x, y]^{T} \mid(x \geq\right.$ $\left.-100 m) \wedge\left(y \geq x \tan \left(30^{\circ}\right)\right) \wedge\left(-y \geq x \tan \left(30^{\circ}\right)\right)\right\}$. It is sufficient to check these parameters for a time horizon of 300 minutes.

Let us denote the discrete state by $z(t)$ and the continuous state vector by $x(t)=$ $\left[s_{x}, s_{y}, v_{x}, v_{y}\right]^{T}$, where $s_{x}$ and $s_{y}$ are the positions in $\mathrm{x}$ - and y-direction, respectively, and $v_{x}$ and $v_{y}$ are the velocities in $\mathrm{x}$ - and $\mathrm{y}$-direction, respectively. The mode approaching is denoted by $z_{1}$, the mode rendezvous attempt by $z_{2}$, and the mode aborting by $z_{3}$. We can formalize the specification as

$$
\begin{gathered}
\text { SR02 } \forall t \in[0,300 \text { min }], \forall x(0) \in \mathcal{X}_{0}:\left(z(t)=z_{2}\right) \Longrightarrow\left(\sqrt{v_{x}^{2}+v_{y}^{2}} \leq 0.055 \mathrm{~m} / \mathrm{s} \wedge\right. \\
\left.\left[s_{x}, s_{y}\right]^{T} \in \mathcal{L}\right) \wedge\left(z(t)=z_{3}\right) \Longrightarrow\left(\left[s_{x}, s_{y}\right]^{T} \notin \mathcal{B}\right) .
\end{gathered}
$$

To solve the above specification, all tools under-approximate the nonlinear constraint $\sqrt{v_{x}^{2}+v_{y}^{2}} \leq 0.055 \mathrm{~m} / \mathrm{s}$ by an octagon as shown in Fig. 3 .

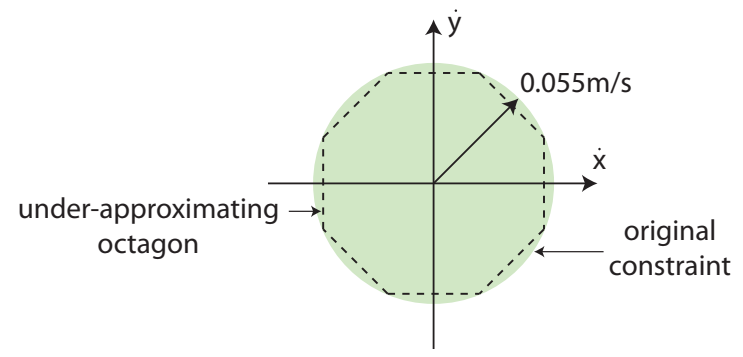

Figure 3: Under-approximation of the nonlinear velocity constraint by an octagon.

Remark on nonlinear constraint In the original benchmark, the constraint on the velocity was set to $0.05 \mathrm{~m} / \mathrm{s}$, but it can be shown that this constraint cannot be satisfied by a counterexample. For this reason, we have relaxed the constraint to $0.055 \mathrm{~m} / \mathrm{s}$.

\subsubsection{Results}

Results of the spacecraft rendezvous benchmark for the $s_{x}-s_{y}$-plane are shown in Fig. 4 for the version SRNA01 and in Fig. 5 for the version SRA01. The computation times of various tools for the spacecraft rendezvous benchmark are listed in Tab. 2.

Note CORA For both benchmark versions CORA was run with a zonotope order of 10 and with a step size of $0.2[\mathrm{~min}]$ for the mode approaching, 0.02 [min] for the mode rendezvous attempt, and 0.2 [min] for the mode aborting (does not exist for version SRNA01). The intersections with the guard sets are calculated with the method of Girard and Le Guernic in [22]. 
In order to find suitable orthogonal directions for the method in [22], we perform the following procedure: first, we project the last zonotope not intersecting the guard set onto the guard set; second, we apply principal component analysis to the generators of the projected zonotope, providing us with the orthogonal directions.

Note Hylaa Hylaa was run with urgent (0-step) transitions disabled, since upon entering the approaching mode, the state may be on the boundary of the line-of-sight cone (strict inequalities are not allowed by linear programming tools and therefore not allowed in Hylaa either).

Note HyDRA We used a time step of 0.1 and support functions with octagonal templates. The time horizon was set to 300 .

Note JuliaReach We used a step size (in dense time) of 0.04 and a one-block partition. The flowpipe was overapproximated with hyperrectangles. We handled discrete transitions by computing the intersection with invariants and guards lazily before overapproximating with a hyperrectangle.

Note SpaceEx SpaceEx was run with the LGG scenario, box directions, and a flowpipe tolerance of 0.2 .

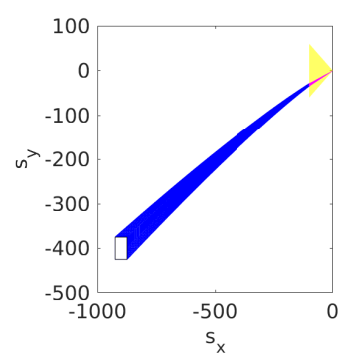

(a) CORA.

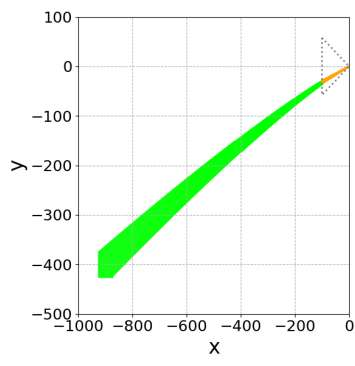

(b) Hylaa.

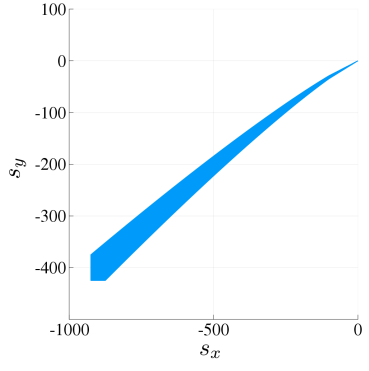

(c) JuliaReach.

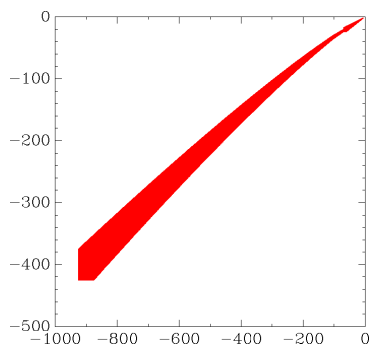

(d) SpaceEx.

Figure 4: Reachable sets for the spacecraft rendezvous benchmark in the $s_{x}-s_{y}$-plane for the benchmark variant without maneuver abortion (SRNA01) 


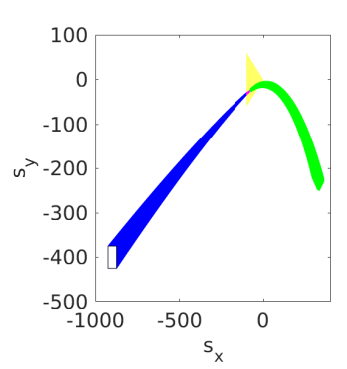

(a) CORA.

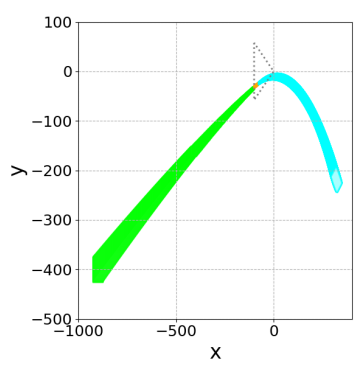

(b) Hylaa.

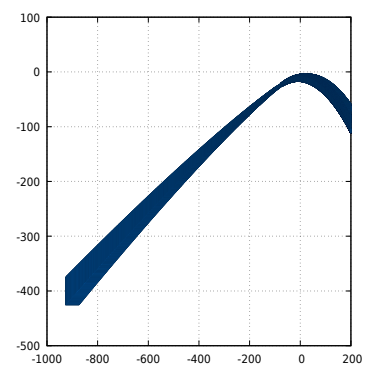

(c) HyDRA.

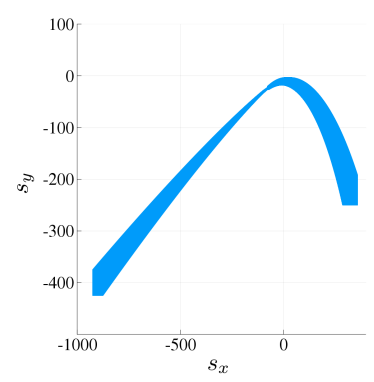

(d) JuliaReach

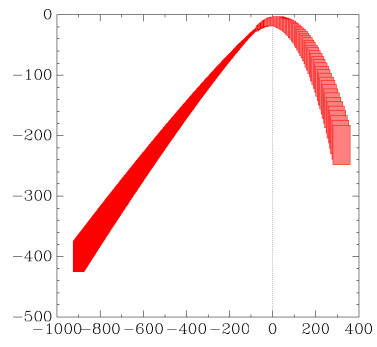

(e) SpaceEx.

Figure 5: Reachable sets for the spacecraft rendezvous benchmark in the $s_{x}-s_{y}$-plane for the benchmark variant with maneuver abortion at $t=120$ [min] (SRA01, over analysis time horizon of 300 [min])

Table 2: Computation Time [s] for the Spacecraft Rendezvous Benchmarks (SR*) for Specification SR02.

\begin{tabular}{|c|c|c|c|c|c|c|c|c|c|c|c|}
\hline tool & NA01 & A01 & A02 & A03 & A04 & A05 & A06 & A07 & A08 & U01 & U02 \\
\hline CORA & 4.7 & 1.4 & 1.2 & 1.7 & 3.6 & 3.7 & 3.7 & 7.8 & 28.5 & 8.3 & 30.6 \\
\hline HyDRA & - & 0.73 & - & - & - & - & - & - & - & - & - \\
\hline JuliaReach & 1.0 & 1.3 & - & - & - & - & - & - & - & 1.8 & 1.7 \\
\hline SpaceEx & 0.33 & 0.31 & - & - & - & - & - & - & - & 0.48 & 2.6 \\
\hline \multicolumn{12}{|c|}{ discrete-time tools } \\
\hline Hylaa & 9.0 & 2.3 & 2.8 & 15 & 30 & 48 & 53 & 15 & 180 & 26 & 489 \\
\hline JuliaReach & 0.5 & 0.6 & - & - & - & - & - & - & - & 0.7 & 0.7 \\
\hline
\end{tabular}




\subsection{Powertrain with Backlash}

\subsubsection{Model}

The powertrain benchmark is an extensible benchmark for hybrid systems with linear continuous dynamics taken from [5, Sec. 6] and [8, Sec. 4]. The essence of this benchmark is recalled here, and the reader is referred to the above-cited papers for more details. The benchmark considers the powertrain of a vehicle consisting of its motor and several rotating masses representing different components of the powertrain, e.g., gears, differential, clutch, among others, as illustrated in Fig. 6. The benchmark is extensible in the sense that the number of continuous states can be easily extended to $n=7+2 \theta$, where $\theta$ is the number of additional rotating masses. The number of discrete modes, however, is fixed and originates from backlash, which is caused by a physical gap between two components that are normally touching, such as gears. When the rotating components switch direction, for a short time they temporarily disconnect, and the system is said to be in the dead zone. The model is available in SpaceEx format on the ARCH website ${ }^{5}$. Discrete-time analysis for the powertrain system should be done with a step size of 0.0005 (5.0E-4).

The set of initial states is

$$
\begin{aligned}
\mathcal{X}_{0} & =\{c+\alpha g \mid \alpha \in[-1,1]\}, \\
c & =[-0.0432,-11,0,30,0,30,360,-0.0013,30, \ldots,-0.0013,30]^{T}, \\
g & =[0.0056,4.67,0,10,0,10,120,0.0006,10, \ldots, 0.0006,10]^{T} .
\end{aligned}
$$

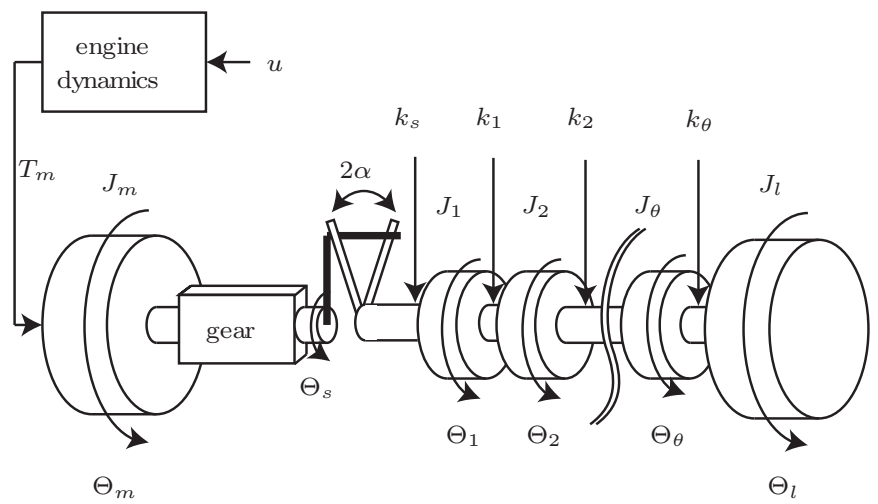

Figure 6: Powertrain model.

\subsubsection{Specifications}

We analyze an extreme maneuver from an assumed maximum negative acceleration that lasts for $0.2[\mathrm{~s}]$, followed by a maximum positive acceleration that lasts for $1.8[\mathrm{~s}]$. The initial states of the model are on a line segment in the $n$-dimensional space. We create different difficulty levels of the reachability problem by scaling down the initial states by some percentage. The

\footnotetext{
${ }^{5}$ cps-vo.org/node/49115
} 
model has the following non-formal specification: after the change of direction of acceleration, the powertrain completely passes the dead zone before being able to transmit torque again. Due to oscillations in the torque transmission, the powertrain should not re-enter the dead zone of the backlash.

To formalize the specification using linear time logic (LTL), let us introduce the following discrete states:

- $z_{1}$ : left contact zone

- $z_{2}:$ dead zone

- $z_{3}$ : right contact zone

For all instances, the common specification is: For all $t \in[0,2], x(0) \in \mathcal{X}_{0},\left(z_{2} U z_{3}\right) \Longrightarrow$ $G\left(z_{3}\right)$. The instances only differ in the size of the system and the initial set, where center $(\cdot)$ returns the volumetric center of a set.

DTN01 $\theta=2, \mathcal{X}_{0}:=0.05\left(\mathcal{X}_{0}-\operatorname{center}\left(\mathcal{X}_{0}\right)\right)+\operatorname{center}\left(\mathcal{X}_{0}\right)$.

DTN02 $\theta=2, \mathcal{X}_{0}:=0.3\left(\mathcal{X}_{0}-\operatorname{center}\left(\mathcal{X}_{0}\right)\right)+\operatorname{center}\left(\mathcal{X}_{0}\right)$.

DTN03 $\theta=2$, no change of $\mathcal{X}_{0}$.

DTN04 $\theta=22, \mathcal{X}_{0}:=0.05\left(\mathcal{X}_{0}-\operatorname{center}\left(\mathcal{X}_{0}\right)\right)+\operatorname{center}\left(\mathcal{X}_{0}\right)$.

DTN05 $\theta=22, \mathcal{X}_{0}:=0.3\left(\mathcal{X}_{0}-\operatorname{center}\left(\mathcal{X}_{0}\right)\right)+\operatorname{center}\left(\mathcal{X}_{0}\right)$.

DTN06 $\theta=22$, no change of $\mathcal{X}_{0}$.

\subsubsection{Results}

Results of the powertrain benchmark in the $x_{1}-x_{3}$-plane are shown in Fig. 7. The computation times of various tools for the powertrain benchmark are listed in Tab. 3.

Note CORA For all benchmark versions CORA was run with a zonotope order of 20 and with a step size of $0.0005 \mathrm{~s}$. The intersections with the guard sets are calculated with the mapping based approach from [5] which avoids the geometric computation of the intersection and therefore scales well with respect to the number of system states.

Note Hylaa Hylaa was run with aggregation turned off. Convex hull aggregation worked, but was slower. The current deaggregation approach in Hylaa did not work well, as overapproximation error seemed to allow states to remain in the dead zone indefinitely, so deaggregation was never triggered.

\subsection{Building Benchmark}

\subsubsection{Model}

This benchmark is quite straightforward: The system is described by $\dot{x}(t)=A x(t)+B u(t)$, $u(t) \in \mathcal{U}, y(t)=C x(t)$, where $A, B, C$ are provided on the ARCH website ${ }^{6}$. The initial set and the uncertain input $\mathcal{U}$ are provided in [27, Tab. 2.2]. Discrete-time analysis for the building system should use a step size of 0.01 . There are two versions of this benchmark:

\footnotetext{
${ }^{6}$ cps-vo.org/node/34059
} 


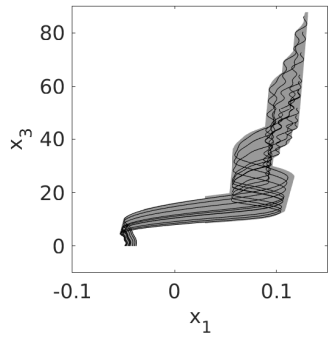

(a) CORA (DTN03).

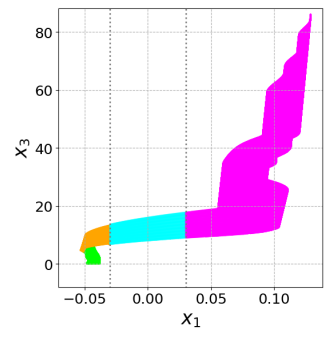

(b) Hylaa (DTN03).

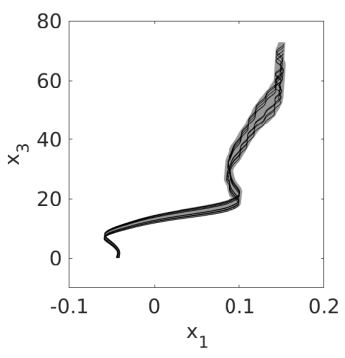

(c) CORA (DTN05).

Figure 7: Reachable sets in the $x_{1}-x_{3}$-plane.

Table 3: Computation Times for the Powertrain Benchmark

\begin{tabular}{|c|c|c|c|c|c|c|c|c|}
\hline \multirow[b]{2}{*}{ tool } & \multicolumn{6}{|c|}{ computation time in $[s]$} & \multicolumn{2}{|c|}{ platform } \\
\hline & DTN01 & DTN02 & DTN03 & DTN04 & DTN05 & DTN06 & language & $\begin{array}{l}\text { machine } \\
\text { (Sec. A) }\end{array}$ \\
\hline CORA & 3.26 & 3.17 & 3.31 & 17.8 & 17.9 & 18.3 & MATLAB & $\mathrm{M}_{\mathrm{CORA}}$ \\
\hline & discrete- & ne tools & & & & & & \\
\hline Hylaa & 6.45 & 15.2 & 49.6 & 12.6 & 43.2 & 154 & Python & $\mathrm{M}_{\text {Hylaa }}$ \\
\hline
\end{tabular}

BLDF01 The inputs can change arbitrarily over time: $\forall t: u(t) \in \mathcal{U}$.

BLDC01 (constant inputs) The inputs are uncertain only in their initial value, and constant over time: $u(0) \in \mathcal{U}, \dot{u}(t)=0$. The purpose of this model instance is to accommodate tools that cannot handle time-varying inputs.

\subsubsection{Specifications}

The verification goal is to check whether the displacement $y_{1}$ of the top floor of the building remains below a given bound. In addition to the safety specification from the original benchmark, there are two UNSAT instances that serve as sanity checks to ensure that the model and the tool work as intended. But there is a caveat: In principle, verifying an UNSAT instance only makes sense formally if a witness is provided (counter-example, under-approximation, etc.). Since most of the participating tools do not have this capability, we run the tools with the same accuracy settings on an SAT-UNSAT pair of instances. The SAT instance demonstrates that the over-approximation is not too coarse, and the UNSAT instance indicates that the over-approximation is indeed conservative.

BDS01 Bounded time, safe property: For all $t \in[0,20], y_{1}(t) \leq 5.1 \cdot 10^{-3}$. This property is assumed to be satisfied.

BDU01 Bounded time, unsafe property: For all $t \in[0,20], y_{1}(t) \leq 4 \cdot 10^{-3}$. This property is assumed to be violated. Property BDU01 serves as a sanity check. A tool should be run with the same accuracy settings on BLDF01-BDS01 and BLDF01-BDU01, returning UNSAT on the former and SAT on the latter. 
Table 4: Computation Times for the Building Benchmark

\begin{tabular}{|c|c|c|c|c|}
\hline \multirow[b]{2}{*}{ tool } & \multicolumn{2}{|c|}{ computation time in $[\mathrm{s}]$} & \multicolumn{2}{|c|}{ platform } \\
\hline & $\begin{array}{c}\text { BLDC01 } \\
\text { BDS01 }\end{array}$ & $\begin{array}{c}\text { BLDF01 } \\
\text { BDS01 }\end{array}$ & language & $\begin{array}{l}\text { machine } \\
\text { (Sec. A) }\end{array}$ \\
\hline CORA & 0.91 & 0.84 & MATLAB & $\mathrm{M}_{\mathrm{CORA}}$ \\
\hline $\mathrm{CORA} / \mathrm{SX}$ & 0.97 & 1.79 & $\mathrm{C}++$ & $\mathrm{M}_{\text {SpaceEx }}$ \\
\hline HyDRA & 0.64 & - & $\mathrm{C}++$ & $\mathrm{M}_{\mathrm{HyDRA}}$ \\
\hline JuliaReach & 0.2 & 0.2 & Julia & $\mathrm{M}_{\text {JuliaReach }}$ \\
\hline SpaceEx & 1.76 & 1.97 & $\mathrm{C}++$ & $\mathrm{M}_{\text {SpaceEx }}$ \\
\hline \multicolumn{5}{|c|}{ discrete-time tools } \\
\hline Hylaa & 1.4 & 11.7 & Python & $\mathrm{M}_{\text {Hylaa }}$ \\
\hline JuliaReach & 0.1 & 0.1 & Julia & $\mathrm{M}_{\text {JuliaReach }}$ \\
\hline
\end{tabular}

BDU02 Bounded time, unsafe property: The forbidden states are $\left\{y_{1}(t) \leq-0.78 \cdot 10^{-3} \wedge t=20\right\}$. This property is assumed to be violated for BLDF01 and satisfied for BLDC01. Property BDU02 serves as a sanity check to confirm that time-varying inputs are taken into account. A tool should be run with the same accuracy settings on BLDF01-BDU02 and BLDC01BDU02, returning UNSAT on the former and SAT on the latter.

\subsubsection{Results}

Results of the building benchmark for state $x_{25}$ over time are shown in Fig. 8-11. The computation times of various tools for the building benchmark are listed in Tab. 4.

Note CORA Since the dynamics of this example is dominated by the input after one second, we use the step size 0.002 for $t \in[0,1]$ and the step size 0.01 for $t \in[1,20]$. The zonotope order is chosen as 100 .

Note HyDRA We use a step size of 0.004 and support functions with an octagonal template as a state set representation.

Note JuliaReach We used the following step sizes (in dense time): 0.003 for BLDF01 and 0.005 for BLDC01. For the property we need to compute (symbolic) reachable states for only a single variable $\left(x_{25}\right)$. We used a three-block partition so that $x_{25}$ is in its own block (the other two blocks contain $x_{1}-x_{24}$ resp. $x_{26}-x_{48}$ ). Furthermore, we decomposed the discretized initial states lazily to achieve higher precision.

Note SpaceEx The accuracy of SpaceEx was set to the largest value possible that satisfies the specification, here $\varepsilon=0.01$. This means the tool can exploit any margin to reduce the number of computations and/or the number of convex sets in the reach set. The resulting, intentional lack of accuracy shows in the plot. 


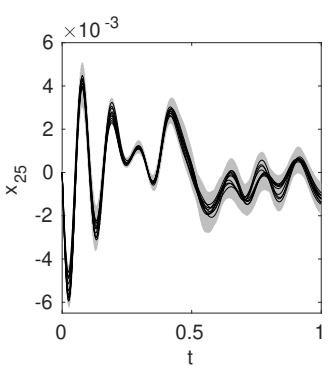

(a) CORA

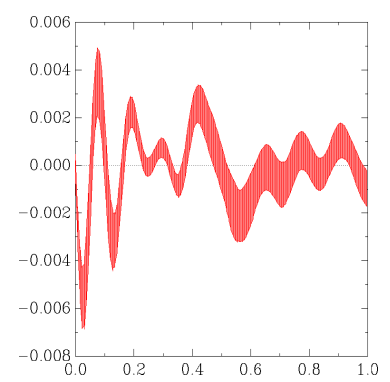

(b) CoraSX.

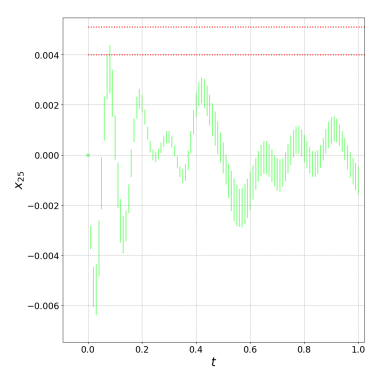

(c) Hylaa.

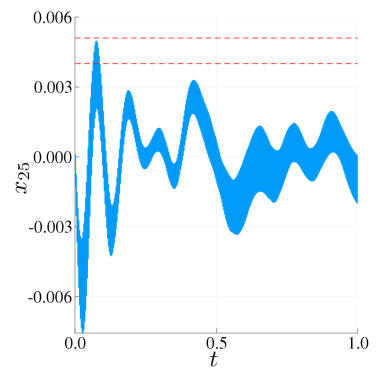

(d) JuliaReach.

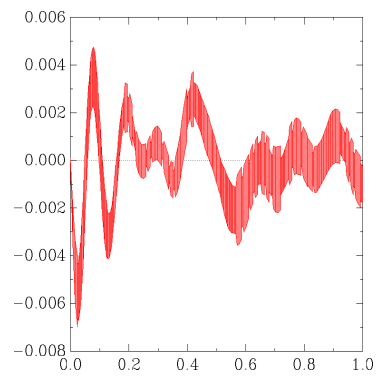

(e) SpaceEx.

Figure 8: Building (BLDF01): Reachable sets of $x_{25}$ plotted over time up to time 1 . Some tools additionally show possible trajectories.

\subsection{Platooning Benchmark}

\subsubsection{Model}

The platooning benchmark considers a platoon of three vehicles following each other. This benchmark considers loss of communication between vehicles. The initial discrete state is $q_{c}$. Three scenarios are considered for the loss of communication:

PLAA01 (arbitrary loss) The loss of communication can occur at any time, see Fig. 12(a). This includes the possibility of no communication at all.

PLADxy (loss at deterministic times) The loss of communication occurs at fixed points in time, which are determined by clock constraints $c_{1}$ and $c_{2}$ in Fig. 12(b). The clock $t$ is reset when communication is lost and when it is re-established. Note that the transitions have must-semantics, i.e., they take place as soon as possible.

PLAD01: $c_{1}=c_{2}=5$.

PLANxy (loss at nondeterministic times) The loss of communication occurs at any time $t \in\left[t_{b}, t_{c}\right]$ in Fig. $12(\mathrm{c})$. The clock $t$ is reset when communication is lost and when it is re-established. Communication is reestablished at any time $t \in\left[0, t_{r}\right]$. This scenario covers loss of communication after an arbitrarily long time $t \geq t_{c}$ by reestablishing communication in zero time.

PLAN01: $t_{b}=10, t_{c}=20, t_{r}=20$. 


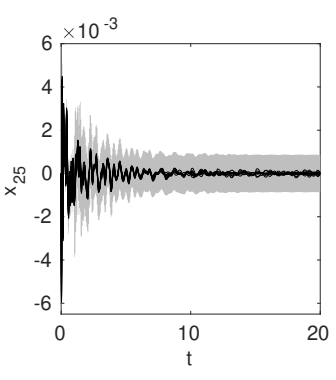

(a) CORA

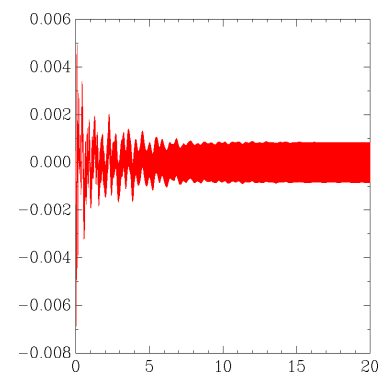

(b) CoraSX.

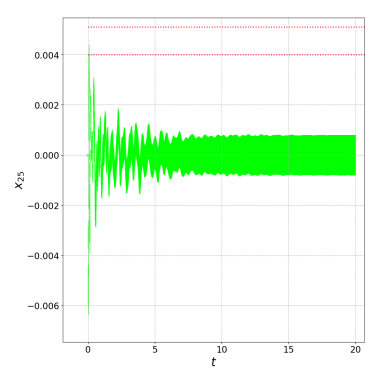

(c) Hylaa.

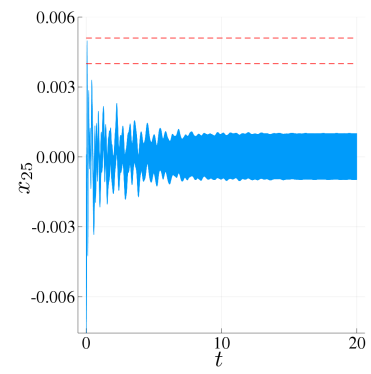

(d) JuliaReach.

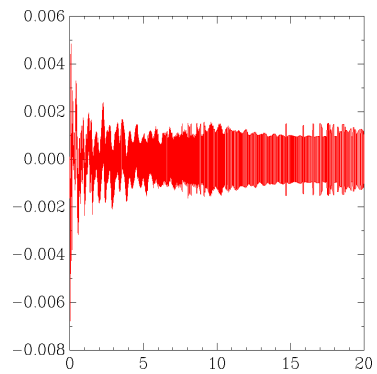

(e) SpaceEx.

Figure 9: Building (BLDF01): Reachable sets of $x_{25}$ plotted over time up to time 20. Some tools additionally show possible trajectories.

The models are available in SpaceEx, KeYmaera, and MATLAB/Simulink format on the ARCH website $^{7}$. Discrete-time analysis for the platoon system should use a step size of 0.1.

Discussion The arbitrary-loss scenario (PLAA) subsumes the other two instances (PLAD, PLAN).

\subsubsection{Specifications}

The verification goal is to check whether the minimum distance between vehicles is preserved. The choice of the coordinate system is such that the minimum distance is a negative value.

BNDxy Bounded time (no explicit bound on the number of transitions): For all $t \in[0,20][\mathrm{s}]$, $x_{1}(t) \geq-d_{\min }[\mathrm{m}], x_{4}(t) \geq-d_{\min }[\mathrm{m}], x_{7}(t) \geq-d_{\min }[\mathrm{m}]$, where $d_{\min }=\mathrm{xy}[\mathrm{m}]$.

BND50: $d_{\min }=50$.

BND42: $d_{\min }=42$.

BND30: $d_{\min }=30$.

UNBxy Unbounded time and unbounded switching: For all $t \geq 0[\mathrm{~s}], x_{1}(t) \geq-d_{\min }[\mathrm{m}]$, $x_{4}(t) \geq-d_{\min }[\mathrm{m}], x_{7}(t) \geq-d_{\min }[\mathrm{m}]$, where $d_{\min }=\mathrm{xy}[\mathrm{m}]$.

UNB50: $d_{\min }=50$.

\footnotetext{
${ }^{7}$ cps-vo.org/node/15096
} 


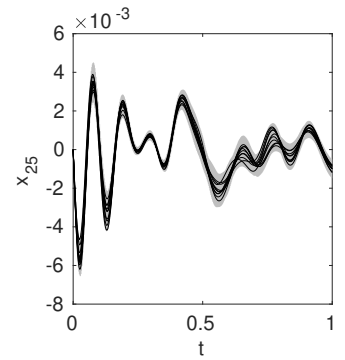

(a) CORA.

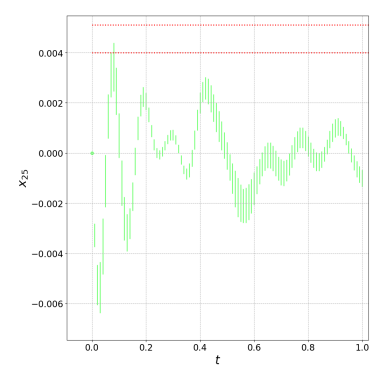

(d) Hylaa

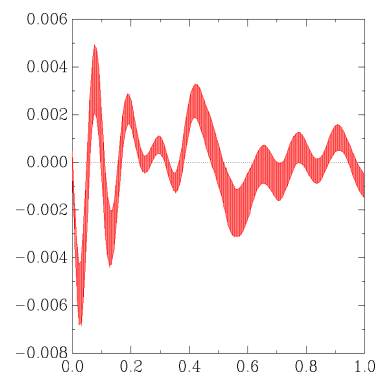

(b) CoraSX.

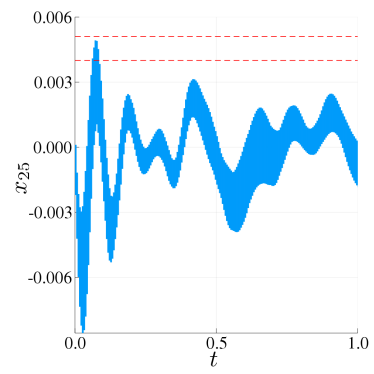

(e) JuliaReach

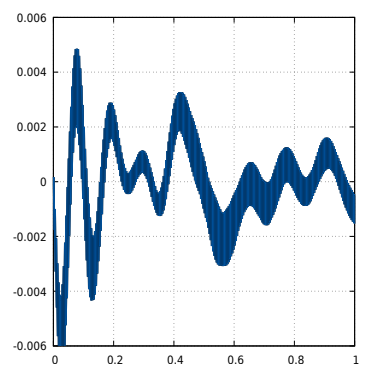

(c) HyDRA.

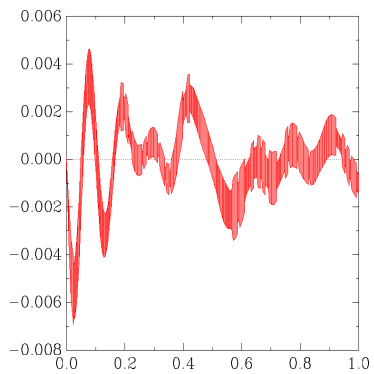

(f) SpaceEx.

Figure 10: Building (BLDC01): Reachable sets of $x_{25}$ plotted over time up to time 1. Some tools additionally show possible trajectories.

UNB42: $d_{\min }=42$.

UNB30: $d_{\min }=30$.

\subsubsection{Results}

Results of the platoon benchmark for state $x_{1}$ over time are shown in Fig. 13-15. The computation times of various tools for the platoon benchmark are listed in Tab. 5 .

Note CORA CORA was run with zonotope order 400 and time step size 0.02 for benchmark version (PLAA01-BND50), with zonotope order 800 and time step size 0.009 for benchmark version (PLAA01-BND42), with zonotope order 20 and time step size $0.02 s$ for benchmark version (PLAD01-BND42), and with zonotope order 200 and time step size 0.02 for benchmark version (PLAD01-BND30). For the unbounded case (PLAN01-UNB50), the reachable set at $t=50$ is increased by $1 \%$ and it is checked when this set is re-entered. Further, zonotope order 400 and time step size 0.01 are used.

For PLAA01, we use the CORA library to rewrite the hybrid automaton as a purely continuous system with uncertain parameters for analysis. This idea is also known as continuization $[6,7]$. After introducing the uncertain matrix

$$
\mathcal{A}=\left\{\alpha A_{c}+\left(1-\alpha A_{n}\right) \mid \alpha \in[0,1]\right\}
$$

we can abstract the model in Fig. 12(a) by

$$
\dot{x}(t) \in \mathcal{A} x \oplus \tilde{\mathcal{U}},
$$




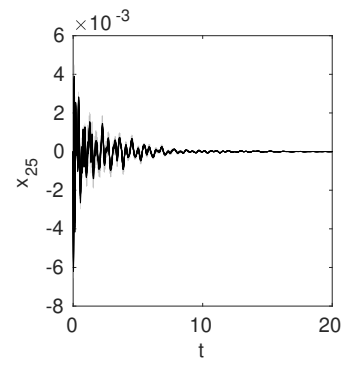

(a) CORA.

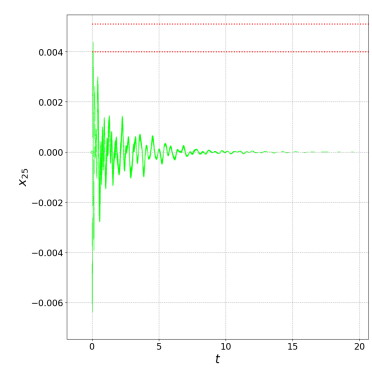

(d) Hylaa.

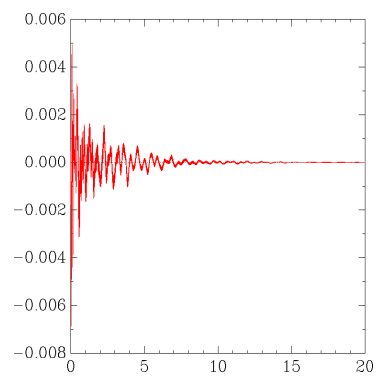

(b) CoraSX.

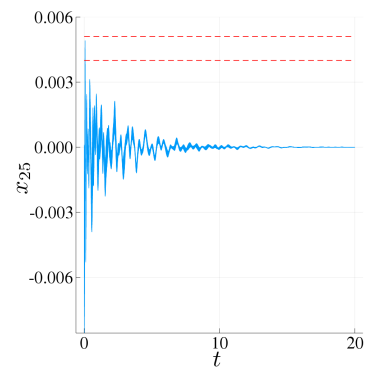

(e) JuliaReach.

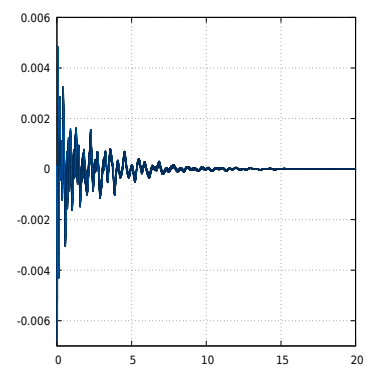

(c) HyDRA.

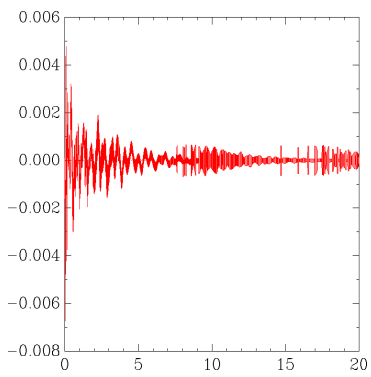

(f) SpaceEx.

Figure 11: Building (BLDC01): Reachable sets of $x_{25}$ plotted over time up to time 20. Some tools additionally show possible trajectories.

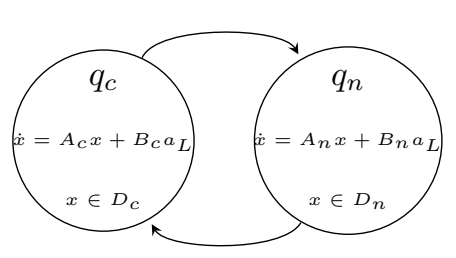

(a) Arbitrary switching.

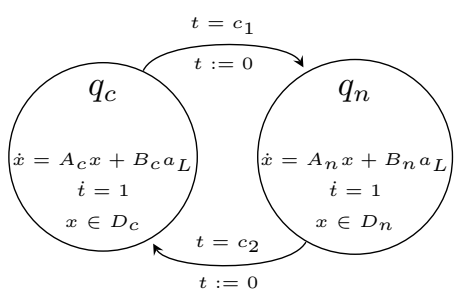

(b) Deterministic switching.

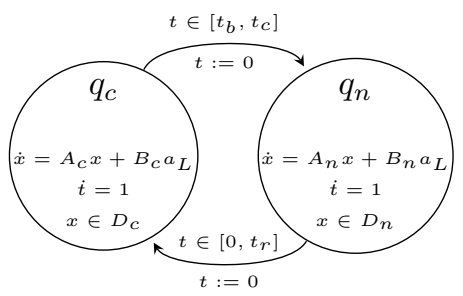

(c) Nondeterministic switching.

Figure 12: Three options adapted from the original benchmark proposal [12]. On the left, the system can switch arbitrarily between the modes. In the middle, mode switches are only possible at given points in time. On the right, mode switches are only possible during given time intervals.

where $\oplus$ denotes the Minkowski addition and $\tilde{\mathcal{U}}=B_{c} \mathcal{U}\left(B_{c}=B_{n}\right)$.

Note HyDRA As HyDRA cannot handle uncertain time-varying inputs we use an instance of the platoon benchmark in which inputs are constant (similar to the building benchmark). To verify instance DBND30 we use a step size of 0.1 and support functions with an octagonal template. For the instance DBND42 we use a step size of 0.33 again with support functions with an octagonal template. 
Note JuliaReach We used the following step sizes (in dense time): 0.01 for PLAD01-BND42, $10^{-5}$ for PLAD01-BND30, and 0.03 for PLAN01-UNB50. In all cases we used a one-block partition and overapproximated the flowpipe with hyperrectangles. We handled discrete transitions by computing the intersection with invariants lazily before overapproximating with a hyperrectangle. In the unbounded-time scenario (PLAD01), termination requires to detect a fixpoint; we check for fixpoints after every discrete transition.

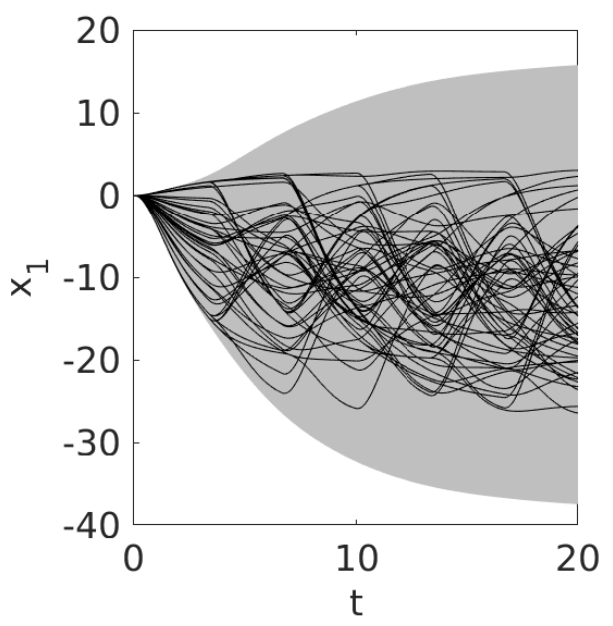

(a) CORA.

Figure 13: PLAA01: Reachable sets of $x_{1}$ plotted over time. CORA additionally shows possible trajectories.

Table 5: Computation Times for the Platoon Benchmark

\begin{tabular}{|c|c|c|c|c|c|c|c|}
\hline \multirow[b]{2}{*}{ tool } & \multicolumn{5}{|c|}{ computation time in $[\mathrm{s}]$} & \multicolumn{2}{|c|}{ platform } \\
\hline & $\begin{array}{c}\text { PLAA01 } \\
\text { BND50 }\end{array}$ & $\begin{array}{c}\text { PLAA01 } \\
\text { BND42 }\end{array}$ & $\begin{array}{c}\text { PLAD01 } \\
\text { BND42 }\end{array}$ & $\begin{array}{c}\text { PLAD01 } \\
\text { BND30 }\end{array}$ & $\begin{array}{c}\text { PLAN01 } \\
\text { UNB50 }\end{array}$ & language & $\begin{array}{l}\text { machine } \\
\text { (Sec. A) }\end{array}$ \\
\hline CORA & 4.17 & 13.7 & 0.12 & 0.33 & 85.4 & MATLAB & $\mathrm{M}_{\mathrm{CORA}}$ \\
\hline HyDRA & - & - & 3.2 & 3.45 & - & $\mathrm{C}++$ & $\mathrm{M}_{\mathrm{HyDRA}}$ \\
\hline JuliaReach & - & - & 0.6 & - & 9.2 & Julia & $M_{\text {JuliaReach }}$ \\
\hline SpaceEx & - & - & 0.63 & 9.6 & 159.2 & $\mathrm{C}++$ & $\mathrm{M}_{\text {SpaceEx }}$ \\
\hline \multirow[t]{2}{*}{ XSpeed } & - & - & 0.57 & 6.23 & 29.41 & $\mathrm{C}++$ & $\mathrm{M}_{\mathrm{XSpeed}}$ \\
\hline & discrete-t & time tools & & & & & \\
\hline Hylaa & - & - & 0.97 & 0.97 & - & Python & $\mathrm{M}_{\text {Hylaa }}$ \\
\hline JuliaReach & - & - & 0.1 & - & 6.4 & Julia & $\mathrm{M}_{\text {JuliaReach }}$ \\
\hline
\end{tabular}




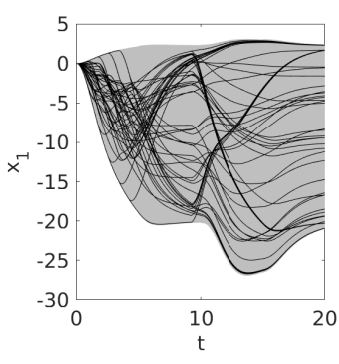

(a) CORA (BND30).

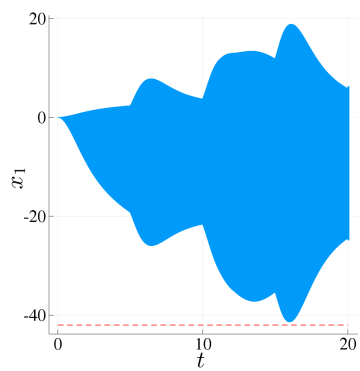

(d) JuliaReach (BND42).

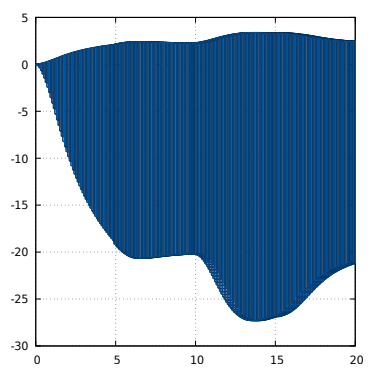

(b) HyDRA (BND30).

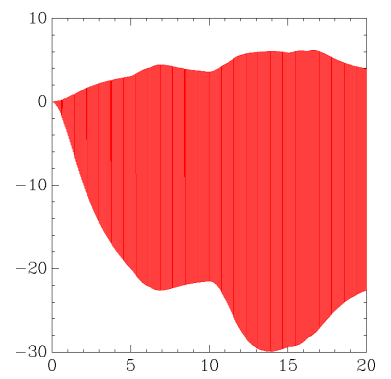

(e) SpaceEx (BND30).

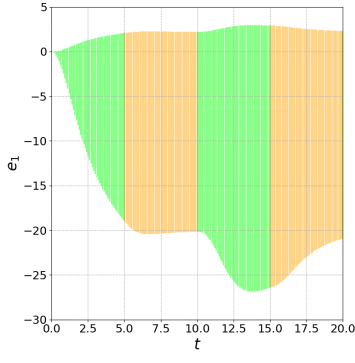

(c) Hylaa (BND30).

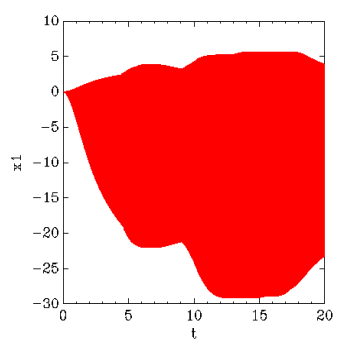

(f) XSpeed (BND30).

Figure 14: PLAD01: Reachable sets of $x_{1}$ plotted over time. Some tools additionally show possible trajectories.

\subsection{Gearbox Benchmark}

\subsubsection{Model}

The gearbox benchmark models the motion of two meshing gears. When the gears collide, an elastic impact takes place. As soon as the gears are close enough, the gear is considered meshed. The model includes a monitor state that checks whether the gears are meshed or free and is available in SpaceEx format ${ }^{8}$ and as a Simulink model ${ }^{9}$. Once the monitor reaches the state meshed, it stays there indefinitely.

With four continuous state variables, the gearbox benchmark has a relatively low number of continuous state variables. The challenging aspect of this benchmark is that the solution heavily depends on the initial state as already pointed out in [17]. For some initial continuous states, the target region is reached without any discrete transition, while for other initial states, several discrete transitions are required.

In the original benchmark, the position uncertainty in the direction of the velocity vector of the gear teeth (x-direction) is across the full width of the gear spline. Uncertainties of the position and velocity in $\mathrm{y}$-direction, which is perpendicular to the $\mathrm{x}$-direction, are considered to be smaller. Due to the sensitivity with respect to the initial set, we consider smaller initial sets. The full uncertainty in x-direction could be considered by splitting the uncertainty in $\mathrm{x}$-direction and aggregating the individual results. For discrete-time analysis of the gearbox system, a step size of 0.0001 (1.0E-4) should be used.

\footnotetext{
${ }^{8}$ cps-vo.org/node/34375

${ }^{9}$ cps-vo.org/node/34374
} 


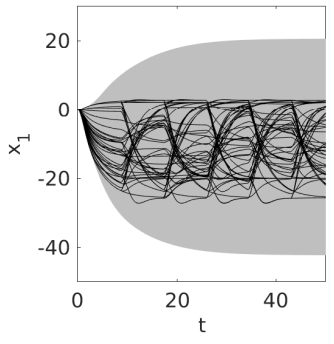

(a) CORA (UNB50).

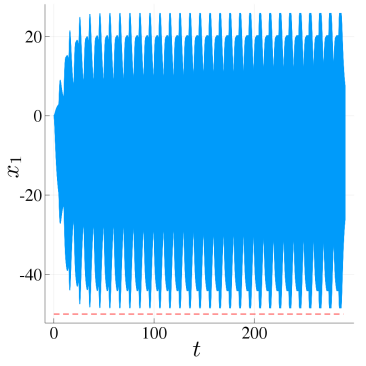

(b) JuliaReach (UNB50).

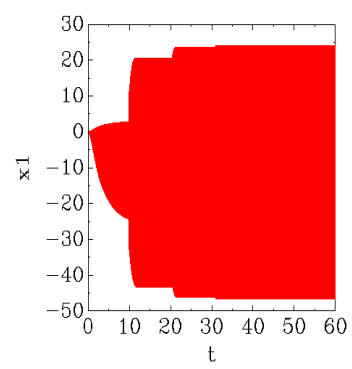

(d) XSpeed (UNB50).

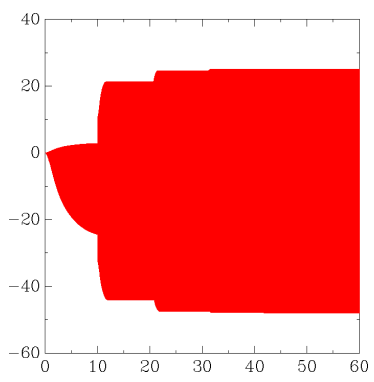

(c) SpaceEx (UNB50).

Figure 15: PLAN01: Reachable sets of $x_{1}$ plotted over time.

GRBX01: The initial set is $\mathcal{X}_{0}=0 \times 0 \times[-0.0168,-0.0166] \times[0.0029,0.0031] \times 0$.

GRBX02: The initial set is $\mathcal{X}_{0}=0 \times 0 \times[-0.01675,-0.01665] \times[0.00285,0.00315] \times 0$.

\subsubsection{Specification}

The goal is to show that the gears are meshed within a time frame of $0.2[\mathrm{~s}]$ and that the bound $x_{5} \leq 20[\mathrm{Nm}]$ of the cumulated impulse is met. Using the monitor states free and meshed, and a global clock $t$, this can be expressed as a safety property as follows: For all $t \geq 0.2$, the monitor should be in meshed. Under nonblocking assumptions, this means that $t<0.2$ whenever the monitor is not in meshed, i.e., when it is in free.

MES01: forbidden states: $($ free $\wedge t \geq 0.2) \vee\left(x_{5} \geq 20\right)$

\subsubsection{Results}

Results of the benchmark for state $x_{3}$ and $x_{4}$ are shown in Fig. 16. The computation times of various tools for the benchmark are listed in Tab. 6.

Note CORA CORA was run with a time step size of $0.0011 \mathrm{~s}$ and a zonotope order of 20 . The intersections with the guard sets were calculated with the method of Girard and Le Guernic [22]. In order to find suitable orthogonal directions for the method in [22], we perform the following procedure: first, we project the last zonotope not intersecting the guard set onto the guard set; second, we apply principal component analysis to the generators of the projected zonotope, providing us with the orthogonal directions. 
Note Hylaa Hylaa used resets to project the system state exactly onto the guard when transitioning from the free mode.

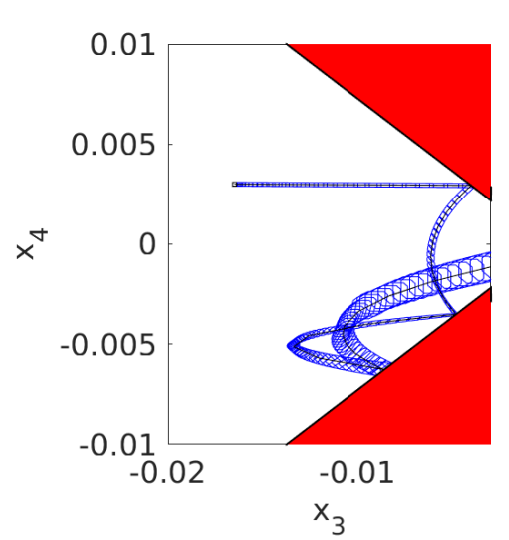

(a) CORA.

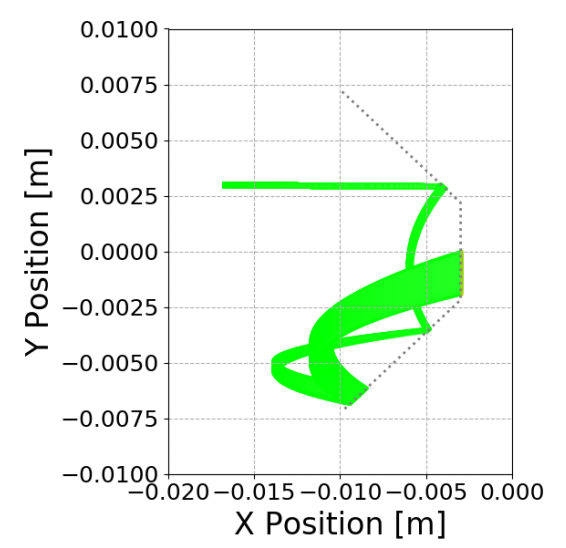

(b) Hylaa.

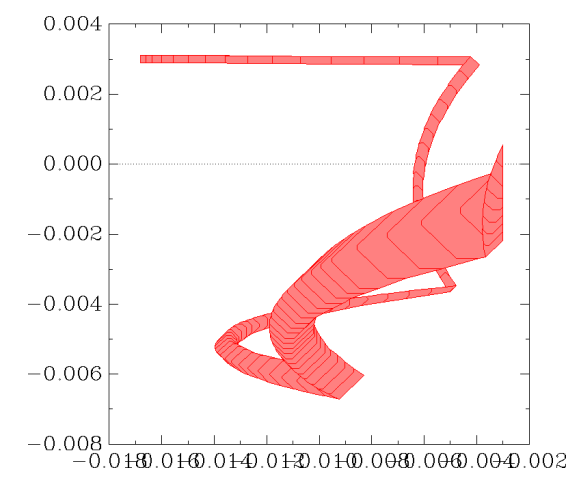

(c) SpaceEx.

Figure 16: Gearbox (GRBX01): Reachable sets of $x_{3}$ and $x_{4}$. Some tools additionally show possible trajectories.

Table 6: Computation Times of the Gearbox Benchmark

\begin{tabular}{lcccccc}
\hline & \multicolumn{2}{c}{ computation time in $[\mathbf{s}]$} & & \multicolumn{2}{c}{ platform } \\
\cline { 2 - 3 } \cline { 5 - 6 } tool & GRBX01-MES01 & GRBX02-MES01 & & language & machine (Sec. A) \\
\hline CORA & 0.36 & 0.48 & & MATLAB & $\mathrm{M}_{\mathrm{CORA}}$ \\
SpaceEx & 0.07 & 0.08 & & $\mathrm{C}++$ & $\mathrm{M}_{\text {SpaceEx }}$ \\
\hline \multirow{3}{*}{ Hylaa } & discrete-time tools & 5.5 & 5.6 & & Python & $\mathrm{M}_{\text {Hylaa }}$ \\
\hline
\end{tabular}




\section{Conclusion and Outlook}

This report presents the results on the third friendly competition for the formal verification of continuous and hybrid systems with linear continuous dynamics as part of the ARCH'19 workshop. The reports of other categories can be found in the proceedings and on the ARCH website: cps-vo.org/group/ARCH.

A major observation of the results is that participating tools have significantly reduced computation times compared to the previous year. Also, Dockerfiles are now available for all tools from gitlab.com/goranf/ARCH-COMP so that running the examples on one's own machine is much easier now. Information about the competition in 2020 will be announced on the ARCH website.

\section{Acknowledgments}

The authors gratefully acknowledge financial support by the European Commission project UnCoVerCPS under grant number 643921, and by DST-SERB, Government of India, under grant number YSS/2014/000623.

\section{A Specification of Used Machines}

\section{A.1 $\mathrm{M}_{\mathrm{CORA}}$}

- Processor: Intel Core i7-7820HQ CPU @ 2.90GHz x 4

- Memory: $32 \mathrm{~GB}$

- Average CPU Mark on www.cpubenchmark.net: 9409 (full), 2027 (single thread)

\section{A.2 $\mathrm{M}_{\mathrm{HyDRA}}$}

- Processor: Intel Core i7-4790K CPU @ 4.00GHz x 8

- Memory: 16 GB

- Average CPU Mark on www.cpubenchmark.net: 11175 (full), 2530 (single thread)

\section{A.3 $\mathrm{M}_{\text {Hylaa }}$}

- Processor: Intel Core i5-5300U @ 2.30GHz x 4

- Memory: $16 \mathrm{~GB}$

- Average CPU Mark on www.cpubenchmark.net: 3755 (full), 1527 (single thread)

\section{A.4 M JuliaReach}

- Processor: Intel Core i5-5200 CPU @ 2.20GHz x 4

- Memory: $8 \mathrm{~GB}$

- Average CPU Mark on www.cpubenchmark.net: 3486 (full), 1397 (single thread) 


\section{A.5 $\mathrm{M}_{\text {SpaceEx }}$}

- Processor: Intel Core i9-8950HK CPU @ $2.9 \mathrm{GHz}$

- Memory: $16 \mathrm{~GB}$

- Average CPU Mark on www.cpubenchmark.net: 14428 (full), 2563 (single thread)

\section{A.6 $\mathrm{M}_{\mathrm{XSpeed}}$}

- Processor: Intel Xeon E5-1650 v4 @ 3.60GHz x 6

- Memory: $64 \mathrm{~GB}$

- Average CPU Mark on www.cpubenchmark.net: 14244 (full), 2182 (single thread)

\section{References}

[1] M. Althoff. An introduction to CORA 2015. In Proc. of the Workshop on Applied Verification for Continuous and Hybrid Systems, pages 120-151, 2015.

[2] M. Althoff, S. Bak, D. Cattaruzza, X. Chen, G. Frehse, R. Ray, and S. Schupp. ARCH-COMP17 category report: Continuous and hybrid systems with linear continuous dynamics. In Proc. of the 4th International Workshop on Applied Verification for Continuous and Hybrid Systems, pages 143-159, 2017.

[3] M. Althoff and D. Grebenyuk. Implementation of interval arithmetic in CORA 2016. In Proc. of the 3rd International Workshop on Applied Verification for Continuous and Hybrid Systems, pages 91-105, 2016.

[4] M. Althoff, D. Grebenyuk, and N. Kochdumper. Implementation of Taylor models in CORA 2018. In Proc. of the 5th International Workshop on Applied Verification for Continuous and Hybrid Systems, pages 145-173, 2018.

[5] M. Althoff and B. H. Krogh. Avoiding geometric intersection operations in reachability analysis of hybrid systems. In Hybrid Systems: Computation and Control, pages 45-54, 2012.

[6] M. Althoff, C. Le Guernic, and B. H. Krogh. Reachable set computation for uncertain time-varying linear systems. In Hybrid Systems: Computation and Control, pages 93-102, 2011.

[7] M. Althoff, A. Rajhans, B. H. Krogh, S. Yaldiz, X. Li, and L. Pileggi. Formal verification of phase-locked loops using reachability analysis and continuization. Communications of the ACM, 56(10):97-104, 2013.

[8] S. Bak, S. Bogomolov, and M. Althoff. Time-triggered conversion of guards for reachability analysis of hybrid automata. In Proc. of the 15th International Conference on Formal Modelling and Analysis of Timed Systems, pages 133-150, 2017.

[9] S. Bak and P. S. Duggirala. Hylaa: A tool for computing simulation-equivalent reachability for linear systems. In Proceedings of the 20th International Conference on Hybrid Systems: Computation and Control, pages 173-178. ACM, 2017.

[10] S. Bak and P. S. Duggirala. Rigorous simulation-based analysis of linear hybrid systems. In International Conference on Tools and Algorithms for the Construction and Analysis of Systems, pages 555-572. Springer, 2017.

[11] S. Bak and P. S. Duggirala. Simulation-equivalent reachability of large linear systems with inputs. In International Conference on Computer Aided Verification, pages 401-420. Springer, 2017.

[12] I. Ben Makhlouf and S. Kowalewski. Networked cooperative platoon of vehicles for testing methods and verification tools. In Proc. of ARCH14-15. 1st and 2nd International Workshop on Applied veRification for Continuous and Hybrid Systems, pages 37-42, 2015. 
[13] Carl Boettiger. An introduction to Docker for reproducible research. ACM SIGOPS Operating Systems Review, 49(1):71-79, 2015.

[14] S. Bogomolov, M. Forets, G. Frehse, K. Potomkin, and C. Schilling. JuliaReach: a toolbox for set-based reachability. In $H S C C, 2019$.

[15] S. Bogomolov, M. Forets, G. Frehse, F. Viry, A. Podelski, and C. Schilling. Reach set approximation through decomposition with low-dimensional sets and high-dimensional matrices. In $H S C C$, pages 41-50, 2018.

[16] N. Chan and S. Mitra. Verifying safety of an autonomous spacecraft rendezvous mission. In Proc. of the 4th International Workshop on Applied Verification of Continuous and Hybrid Systems, pages 20-32, 2017.

[17] H. Chen, S. Mitra, and G. Tian. Motor-transmission drive system: a benchmark example for safety verification. In Proc. of ARCH14-15. 1st and 2nd International Workshop on Applied veRification for Continuous and Hybrid Systems, pages 9-18, 2015.

[18] WH Clohessy. Terminal guidance system for satellite rendezvous. Journal of the Aerospace Sciences, 27(9):653-658, 1960.

[19] G. Frehse. Reachability of hybrid systems in space-time. In Alain Girault and Nan Guan, editors, Proc. Int. Conf. Embedded Software, EMSOFT, Amsterdam, Netherlands, October 4-9, 2015, pages 41-50. IEEE, 2015.

[20] G. Frehse, C. Le Guernic, A. Donzé, S. Cotton, R. Ray, O. Lebeltel, R. Ripado, A. Girard, T. Dang, and O. Maler. SpaceEx: Scalable verification of hybrid systems. In Proc. of the 23rd International Conference on Computer Aided Verification, LNCS 6806, pages 379-395. Springer, 2011.

[21] G. Frehse, R. Kateja, and C. Le Guernic. Flowpipe approximation and clustering in space-time. In C. Belta and F. Ivancic, editors, Proc. Int. Conf. Hybrid systems: computation and control, HSCC, April 8-11, 2013, Philadelphia, PA, USA, pages 203-212. ACM, 2013.

[22] A. Girard and C. Le Guernic. Zonotope/hyperplane intersection for hybrid systems reachability analysis. In Proc. of Hybrid Systems: Computation and Control, LNCS 4981, pages 215-228. Springer, 2008.

[23] A. Gurung, A. Deka, E. Bartocci, S. Bogomolov, R. Grosu, and R. Ray. Parallel reachability analysis for hybrid systems. In ACM/IEEE International Conference on Formal Methods and Models for System Design, MEMOCODE, pages 12-22. IEEE, 2016.

[24] R. Ray and A. Gurung. Poster: Parallel state space exploration of linear systems with inputs using xspeed. In Proc. of HSCC'15, pages 285-286. ACM, 2015.

[25] R. Ray, A. Gurung, B. Das, E. Bartocci, S. Bogomolov, and R. Grosu. XSpeed: Accelerating reachability analysis on multi-core processors. In Proc. of $H V C$ 2015, volume 9434 of $L N C S$, pages 3-18, 2015.

[26] S. Schupp, E. Abraham, I. Ben Makhlouf, and S. Kowalewski. HyPro: A C++ library for state set representations for hybrid systems reachability analysis. In Proc. NFM'17, volume 10227 of LNCS, pages 288-294. Springer, 2017.

[27] H.-D. Tran, L. V. Nguyen, and T. T. Johnson. Large-scale linear systems from order-reduction. In Proc. of ARCH16. 3rd International Workshop on Applied Verification for Continuous and Hybrid Systems, pages 60-67, 2017. 